\title{
MÉTODOS DE FRACIONAMENTO DA CORTADA DO VOLEIBOL SOB A PERSPECTIVA DE PROFESSORES DE EDUCAÇÃO FÍSICA
}

\author{
Raquel Maia Bokums \\ Universidade Paulista, São Paulo, São Paulo, Brasil
}

Marcelo Massa

Universidade de São Paulo, São Paulo, São Paulo, Brasil

Jaqueline Freitas Neiva

Universidade de São Paulo, São Paulo, São Paulo, Brasil

Jusselma Ferreira Maia

Universidade Paulista, São Paulo, São Paulo, Brasil

Maria Teresa Marques

Universidade de São Paulo, São Paulo, São Paulo, Brasil

\section{Cassio Miranda Meira Jr.}

Universidade de São Paulo, São Paulo, São Paulo, Brasil

\section{Resumo}

Pelo fato de o fracionamento da prática da cortada ser um tema polêmico que tem gerado inconsistências teóricas e práticas, o objetivo do presente estudo foi investigar quais métodos de fracionamento da cortada são utilizados por professores de Educação Física em diferentes fases profissionais no processo ensino-aprendizagem da cortada. Além disso, pretendeu-se descrever as justificativas dos professores para a escolha dos métodos. A amostra foi composta por 36 professores de Educação Física com níveis distintos de experiência na área, os quais responderam a um questionário sobre o uso de métodos de ensino relativos ao fracionamento da cortada do voleibol. As maiores frequências de respostas favoráveis ao fracionamento foram fornecidas por professores em estágios iniciais da carreira, os quais alegaram que a complexidade da cortada é o motivo principal para o uso de tal procedimento.

Palavras-chave: Métodos. Ensino. Voleibol. Educação Física e Treinamento.

\section{Introdução}

Tabilidades motoras específicas das modalidades esportivas são - conteúdos legítimos da Educação Física (EF) que devem ser transmitidos ao longo do processo educacional. Uma decisão importante a ser tomada pelo professor no processo de ensino-aprendizagem de habilidades motoras é o método de ensino a ser adotado. A maximização do ensino deve ser objetivo do professor e é por intermédio 
do método adotado, mesmo que inconscientemente, que o professor facilita a condução do aluno aos meios mais efetivos de execução de habilidades. Portanto, a escolha do método de ensino deve ser uma preocupação constante do professor, uma vez que lançando mão do meio mais adequado de facilitação pedagógica (método), a prática profissional e o processo ensino-aprendizagem serão fundamentados e transcorrerão com maior confiança e segurança (TANI, 1996).

A qualidade da prática profissional no ensino de diferentes habilidades motoras carrega influências do nível de experiência do professor (JANUÁRIO, 1996). Espera-se, assim, que um professor bem formado em sua graduação, atualizado, comprometido com a sua prática e com mais experiência na atuação docente desenvolva o processo ensino-aprendizagem de habilidades motoras com maior fundamentação teórica e prática. Assim, a experiência profissional costuma ser definida em função do tempo na profissão. Huberman (1992) estabeleceu as seguintes fases ou estágios da vida profissional do docente: 1) entrada: de um a três anos de carreira (fase inicial); 2) estabilização: de quatro a seis anos de carreira (fase de consolidação); 3) serenidade: de sete a 25 anos de carreira (fase de distanciamento afetivo); 4) desinvestimento: de 30 a 40 anos de carreira (fase final). Portanto, pode-se avançar a hipótese que dependendo da fase em que se encontra o professor, a utilização de métodos de ensino de habilidades motoras pode variar. A natureza seriada das habilidades motoras implica na execução de uma sequência de um padrão de movimentos em um padrão espacial e temporal adequado para que a meta seja alcançada (PUBLIO; TANI, 1993). Por exemplo, para que uma cortada do voleibol seja realizada por meio de um padrão espacial e temporalmente organizado em relação à demanda do ambiente, é preciso executar sequencialmente os componentes de posição de expectativa, passadas de aproximação, salto, posicionamento dos membros superiores, ataque à bola, finalização e aterrissagem. No presente estudo, o tipo de método de ensino a ser enfatizado refere-se ao fracionamento (ou não) de habilidades motoras.

Esse tema tem origem no estudo de Naylor e Briggs (1963), que propuseram os conceitos de "complexidade" e "organização" como critérios para decidir a prática mais efetiva a ser utilizada na aprendizagem de listas cognitivas. Complexidade é definida como o número de elementos de uma tarefa, em função das várias partes que a compõem e das solicitações do processamento de informação. Organiza- 
ção refere-se à interação/interdependência entre os elementos da tarefa. A decisão sobre qual prática motora escolher depois de avaliar a habilidade-alvo nos critérios de complexidade e organização deve ser tomada da seguinte forma: prática das partes para tarefas com alta complexidade e baixa organização (por exemplo, um salto ornamental); esse tipo de prática é desenvolvido com tentativas isoladas, separadas e fracionadas de cada elemento da tarefa, os quais serão resgatados e unidos posteriormente. Por sua vez, prática do todo para tarefas com baixa complexidade e alta organização (por exemplo, um saque); esse tipo de prática é desenvolvido com todas as tentativas do processo sendo praticadas integralmente de modo que todos os elementos da tarefa são executados sequencialmente como um todo. Para tarefas motoras com níveis médios de complexidade e organização ou de difícil classificação em relação a esses dois critérios - sugere-se o uso de práticas mistas, ou seja, a combinação de prática do todo e das partes (MAGILL, 2011; SCHMIDT; WRISBERG, 2008; WRISBERG, 2007). Esses pressupostos teóricos fornecem sugestões para a escolha do método de ensino e permitem deduzir ou antecipar os efeitos do método no processo ensino-aprendizagem de habilidades motoras. Vários estudos têm fornecido suporte a esses pressupostos na investigação de habilidades motoras do judô (GOMES, 2007; MURRAY, 1981), do futebol (CORRÊA; SILVA; PAROLI, 2004), da ginástica artística (BAECKER, 1984; PUBLIO; TANI, 1993), da natação (HIDD, 1991), do voleibol, especificamente a cortada (DUTRA et al., 2006b; DUTRA et al., 2006a; KRUG; KRUG, 1983), e de outras modalidades (para uma revisão sistemática, cf. FONTANA et al., 2009).

A considerar que a aquisição de habilidades motoras é objeto comum no contexto de Aprendizagem Motora e no contexto de EF escolar, o fracionamento da prática tem sido um tema integrador dessas duas subáreas de interesse. No fundo, entendemos que, na abordagem do tema, ambas as subáreas preocupam-se com o mesmo fenômeno, isto é, a melhor forma de aprender habilidades motoras. No presente estudo, denominaremos o meio de aquisição ou a estratégia pedagógica de "método de ensino", já que o contexto-alvo é o da docência escolar. Nesse ambiente particular, a prática do todo é denominada "método do todo ou global". Por sua vez, a prática das partes é denominada "método parcial, analítico ou partes". Caso as partes sejam praticadas isoladamente e então adicionadas em sequências maiores 
até que a tarefa completa seja praticada, tem-se o "método parcial progressivo ou por partes progressivas", "de adição" ou "de segmentação" (BAECKER, 1984; HIDD, 1991; KNAPP, 1970; XAVIER, 1986; WRISBERG, 2007). Existe ainda o "método misto", composto pelo uso de uma sequência cíclica combinada, todo-parte-todo, em que o conteúdo de partes mais difíceis é desenvolvido primeiro isoladamente, para que depois sejam agrupadas no todo.

Com o aumento da popularidade do voleibol nas últimas décadas, as habilidades motoras específicas desse esporte vêm despertando grande interesse em alunos e professores de EF. A cortada, especificamente, tem sido apontada por professores, técnicos e pesquisadores do voleibol como o fundamento de maior complexidade de aprendizado e de maior dificuldade de execução (BIZZOCHI, 2000; BEZAULT, 2002; MACHADO, 2006; CASCO, 2007). Pode-se definir a cortada como uma habilidade de alta exigência tanto em termos de complexidade, como de organização. Os componentes da cortada podem ser definidos como movimentos discretos - em que é possível a identificação de início e fim (MAGILL, 2011) - e se apresentam sequencialmente da seguinte forma: posição de expectativa (A), passadas de aproximação (B), salto (C), posicionamento dos membros superiores (D), ataque à bola $(\mathrm{E})$, finalização $(\mathrm{F})$ e aterrissagem $(\mathrm{G})$. Por isso, teoricamente, o método misto seria o mais indicado para facilitar o processo ensino-aprendizagem de uma habilidade como essa, em que a sequência "A-B-C-D-E-F-G" possui muitos elementos e alto grau de interdependência entre eles (MAGILL, 2011; NAYLOR; BRIGGS, 1963; SCHMIDT; WRISBERG, 2008; WRISBERG, 2007). No entanto, os estudos de Dutra, Paolucci, Leite, Fialho, Xavier \& Benda (2006) e Dutra et al. (2006a) indicaram ausência de diferenças entre os métodos misto, do todo e das partes. Ainda, o estudo de Krug \& Krug (1983) evidenciou que o método das partes mostrou ser mais efetivo que o do todo e que o método misto revelou-se neutro.

Em suma, se, por um lado, os estudos que investigaram o fracionamento no processo ensino-aprendizagem da cortada apontam levemente para o uso do método das partes, por outro, a aplicação dos critérios de complexidade e organização propostos por Naylor e Briggs (1963) sugere o uso de métodos mistos. Assim, entendemos que essas inconsistências encontradas na literatura podem ser refletidas na prática de professores de EF quando enfrentam a necessidade de escolher um método de ensino. O objetivo do presente estudo foi 
investigar quais métodos de fracionamento da cortada são utilizados por professores de Educação Física em diferentes fases profissionais no processo ensino-aprendizagem da cortada. Além disso, pretendeuse descrever as justificativas dos professores para a escolha dos métodos.

\section{Método}

Amostra

Trinta e seis professores de EF, ativos, provenientes de instituições públicas e particulares localizadas na Grande São Paulo, foram selecionados e entrevistados por conveniência. A amostra foi composta por $66 \%$ de professores do gênero masculino e $34 \%$ do feminino. Por razões operacionais (JANUÁRIO, 1996), o critério adotado para definir o tempo de experiência dos professores foi o de Huberman (1992). A amostra, portanto, foi classificada de acordo com três grupos em relação à fase da carreira docente em EF: Grupo 1 (G1): 12 professores iniciantes com até 3 anos experiência (fase de entrada); Grupo 2 (G2): 12 professores com 4 a 6 anos de experiência (fase de estabilização); Grupo 3 (G3): 12 professores experientes entre 7 e 25 anos de experiência (fase de serenidade). A variável "gênero" foi contrabalanceada nos grupos.

Instrumento e Procedimentos

Optou-se pela aplicação de um questionário semi-estruturado, com perguntas abertas e fechadas, por meio das quais o professor teve a possibilidade de discorrer sobre o tema proposto. $\mathrm{O}$ questionário foi composto por três partes, contendo seis perguntas elaboradas a partir de dados conceituais provenientes da literatura sobre voleibol (BOJIKIAN, 1999; CARVALHO, 1980; MACHADO, 2006). A primeira parte contemplou dados pessoais a fim de caracterizar os professores nas variáveis gênero, local de atuação e tempo de experiência. A segunda parte foi composta por duas questões acerca de fundamentos do voleibol, apenas para confirmar o conhecimento sobre o voleibol. Por fim, a (terceira) parte principal envolveu duas questões, uma de característica fechada sobre qual método de ensino da cortada do voleibol utiliza (todo, partes, partes progressivas e misto) e outra de natureza aberta, sobre o porquê da escolha do método.

Pensar a Prática, Goiânia, v. 16, n. 3, p. 619-955, jul./set. 2013

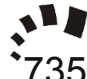


Os professores responderam ao questionário de forma voluntária, alguns tendo enviado as respostas por correio eletrônico e outros por entrega direta aos pesquisadores. A coleta de dados foi realizada ao longo de três meses. Cada professor respondeu às questões de forma individual.

Análise dos Dados

As frequências de respostas dos professores acerca dos métodos utilizados foram organizadas em uma tabela de contingência e comparadas por meio de testes qui-quadrado simples (THOMAS; NELSON; SILVERMAN, 2012). O nível de significância foi estabelecido em 5\%. As respostas principais fornecidas sobre o motivo do emprego dos métodos foram condensadas em um quadro-síntese.

\section{Resultados}

Na Tabela 1, encontram-se as frequências à questão sobre qual método de ensino foi o mais utilizado no ensino da cortada, estratificadas por fases de carreira docente em Educação Física.

Tabela 1: Tabela de contingência referente aos métodos de ensino utilizados pelos professores de Educação Física no ensino da cortada em cada fase da carreira docente.

\begin{tabular}{|c|c|c|c|c|}
\hline & Entrada & Estabilização & Serenidade & Total \\
\hline Todo & $2(16,6 \%)$ & $1(8,3)$ & 0 & $3(8,3 \%)$ \\
\hline Partes & 0 & $2(16,6 \%)$ & $2(16,6 \%)$ & $4(11,1 \%)$ \\
\hline Partes Progressivas & $8(66,7 \%)^{*}$ & $5(41,7 \%)$ & $4(33,4 \%)$ & $17(47,2 \%)^{*}$ \\
\hline Misto & $2(16,6 \%)$ & $4(33,4 \%)$ & $6(50 \%)$ & $12(33,4 \%)^{*}$ \\
\hline Total & 12 & 12 & 12 & 36 \\
\hline
\end{tabular}

* $p<0,05$ (diferença significativa em relação aos outros valores da coluna)

$\mathrm{Na}$ estratificação por grupos, foi detectada diferença significativa pelo teste estatístico qui-quadrado no G1 (Fase de entrada - 1 a 3 anos de experiência) [X2=6,00; graus de liberdade $=2 ; \mathrm{p}=0,049]$, com o método por partes progressivas tendo apresentado maior frequência de respostas quando comparado aos outros métodos. Em relação à análise sem estratificação, o teste estatístico também revelou diferença significativa $[\mathrm{X} 2=14,89$; graus de liberdade $=3 ; \mathrm{p}=0,002]$, com os métodos por partes progressivas e misto sendo mais utilizados do que os métodos do todo e das partes. 


\begin{tabular}{|c|l|}
\hline Método & \multicolumn{1}{|c|}{ Razões } \\
\hline Todo & $\begin{array}{l}\text { Por ser uma habilidade que possui componentes muito relacionados, a } \\
\text { aprendizagem é mais facilitada. Global devido que a criança no seu } \\
\text { processo de desenvolvimento intelectual e motor, necessita de um arranjo } \\
\text { motor muito extenso, para que com isso ele consiga ter um } \\
\text { desenvolvimento motor adequado para o seu processo de aprendizagem. Eu } \\
\text { entendo que a cortada é um movimento complexo, no qual a interação dos } \\
\text { elementos e tão forte que ao separar para ensiná-los descaracterizamos a } \\
\text { cortada e o aluno tem maior dificuldade de aprender. }\end{array}$ \\
\hline Partes & $\begin{array}{l}\text { Serve para obter coordenação das partes para depois realizar o movimento } \\
\text { completo. O ensino se torna mais fácil de entender e de executar, partindo } \\
\text { daí para o global juntando as partes. Ensino o gesto motor por partes, } \\
\text { primeiro a passada/alavanca/salto do ataque/ movimento do braço /encaixe } \\
\text { da mão na bola. }\end{array}$ \\
\hline Progressivas & $\begin{array}{l}\text { Possibilita a prática isolada dos movimentos, ajudando o aluno a fixar o } \\
\text { fundamento, para que gradativamente, observando melhor os erros, as } \\
\text { outras partes sejam acrescentadas. É uma habilidade que apresenta muita } \\
\text { informação e muitos graus de liberdade para o aluno tentar controlar de uma } \\
\text { só vez, por isso, é importante introduzir os componentes aos poucos, sem } \\
\text { perder a relação que há entre eles. Por se tratar de um fundamento que } \\
\text { considero com um grau de dificuldade elevado, acredito que dividindo o } \\
\text { movimento ajudará no resultado final (simples para complexo). }\end{array}$ \\
\hline Misto & $\begin{array}{l}\text { A compreensão corporal durante a execução do gesto é melhor entendida } \\
\text { através do parcionamento do mesmo, contudo é a partir da dinâmica do jogo } \\
\text { que se aprimora o desempenho, ou seja, o método misto auxilia tanto na } \\
\text { eficácia quanto na eficiência do gesto. O seu ensino deverá ser realizado } \\
\text { inicialmente separadamente; todavia a sua aplicação requer uma prática } \\
\text { contextualizada em interligação com o } 1^{\circ} \text { e } 2^{\circ} \text { toque, na medida em que os } \\
\text { tempos de saída para o ataque estão dependentes das características destas } \\
\text { ações (no } 1^{\circ} \text { toque recepção ou defesa e no } 2^{\circ} \text { toque o levantamento). Nesse } \\
\text { sentido, esses aspectos devem ser de seguida integrados. }\end{array}$ \\
\hline
\end{tabular}

Quadro 1: Síntese das razões principais pelas quais os professores utilizaram os métodos de ensino.

\section{Discussão}

Os resultados indicaram que os professores fizeram uso mais frequente do método por partes progressivas e do método misto, fato que fornece suporte aos pressupostos teóricos que sugerem combinação do método do todo com o método das partes quando a tarefa-alvo possui alta complexidade e alta organização - tal qual a cortada (MAGILL, 2011; NAYLOR; BRIGGS，1963; SCHMIDT; WRISBERG，2008; WRISBERG, 2007). A utilização do fracionamento em algum grau mesmo que mínimo - também corrobora o padrão de resultados de estudos empíricos que investigaram o tema no processo ensino-aprendizagem da cortada (DUTRA et al., 2006b; DUTRA et al., 2006a; KRUG; KRUG, 1983). Portanto, parece que a recomendação que se pode fazer é evitar o método do todo no ensino-aprendizagem da cor- 
tada. Mesmo que a cortada enseje interação e interdependência entre componentes (por exemplo, transição das passadas de aproximação para o salto, e salto seguido de posicionamento dos membros superiores e ataque à bola) - e isso remete ao uso da prática do todo -, pesquisadores e professores parecem concordar com utilização de métodos de fracionamento. Ainda, talvez se possa especular que para habilidades-alvo com alta complexidade e alta organização, a melhor opção seja pela introdução de algum tipo de fracionamento ao longo da prática.

As justificativas dos professores em início de carreira para a utilização mais frequente do método por partes progressivas podem ser resumidas da seguinte maneira: "a cortada é um complexo agregado de unidades e então se deve enfatizar o acúmulo gradativo e combinado das várias fases para depois enfatizar o movimento completo". A estratégia de fracionamento ainda foi defendida pelos professores, pois proporciona "rápida melhora técnica" e "progressão estável na melhora física". As desvantagens do fracionamento seriam, na opinião dos professores, "desmotivação", "ausência de uma participação ampla da coordenação e dos sistemas circulatórios e respiratórios" e "dificuldade para decidir sobre o que dever ser selecionado como parte ou fase da habilidade motora".

Em momentos mais tardios da carreira docente em EF - fases de estabilização e serenidade - os professores tenderam a utilizar mais o método misto de combinação do todo e das partes em detrimento do método por partes progressivas. Esse padrão está de acordo com a literatura que aponta diferenças entre profissionais iniciantes e experientes na resolução de problemas e no planejamento de aulas (MOREIRA; PEREIRA, 2008). Parece então que estar em uma fase distinta da carreira implica em alterar os meios pedagógicos de professores de EF. O professor com mais anos de experiência parece não dedicar tanto tempo a técnicas mais demoradas - tal qual o fracionamento -, pois possui maior estoque de estratégias acerca "do que fazer quando" de modo que leva menos tempo para resolver um problema e, quando resolve, o faz detalhadamente, talvez se esmerando mais na condução de atividades desenvolvidas pelo todo. Professores iniciantes, por sua vez, levam mais tempo para resolver um problema, e quando resolvem, o fazem imediatamente (COSTA, 1996). Professores iniciantes também são mais inseguros, em relação aos experientes, quanto ao planejamento de aulas (MOREIRA; PEREIRA, 2008). Ou- 
tras características diferentes entre iniciantes e professores na docência são (BERNILER, 1986): experientes evidenciam maiores capacidades cognitivas e de autorregulação, constroem competência de forma gradual e durante um longo período de tempo.

Os motivos apontados pelos professores para o uso do método misto foi por causa da maior possibilidade de correção dos erros de execução, do domínio da técnica e, principalmente, por causar melhor motivação. Um número muito pequeno de professores - $8 \%$ - citou a utilização do método do todo. Esses poucos alegaram que usam esse método porque uma das principais características da cortada é a sincronização com a bola. Argumentaram ainda que a cortada apresenta muitos componentes relacionados entre si e, por isso, o fracionamento das partes poderia descaracterizar o gesto motor. Esses argumentos são bastante convincentes quando se considera, segundo Naylor e Briggs (1963), a "organização" da habilidade motora. O critério de "complexidade" pareceu ser ignorado por esses professores. Segundo os autores supracitados, o método do todo seria mais efetivo para habilidades de alta organização e baixa complexidade, o que não é o caso da cortada.

Da mesma forma, um número reduzido de professores - $11 \%$ - relatou o emprego do método das partes. Esses resultados fornecem suporte aos estudos de Canfield (1981) e Canfield e Reis (1998), segundo os quais a aprendizagem da cortada deve ocorrer pelo método das partes. A principal razão citada para a escolha desse método foi que, primeiro, o aluno deve adquirir coordenação dos movimentos separadamente para depois realizar o movimento completo, caso contrário, terá mais dificuldades para a finalização da habilidade motora; o uso do método das partes, inicialmente, torna mais fácil o entendimento e execução dos movimentos, para que depois se possa lançar mão do método do todo. O método das partes é, no fundo, uma questão de transferência de aprendizagem, isto é, como os elementos praticados isoladamente irão se transferir quando forem integrados à série como um todo. Nesse contexto, a transferência da parte para o todo parece ser mais efetiva para tarefas seriadas de longa duração, quando as ações e os erros de um elemento não afetam significativamente as ações do elemento subsequente, isto é, em habilidades de alta complexidade e baixa organização (MAGILL, 2011; PUBLIO; TANI, 1993; SCHMIDT; WRISBERG, 2008). Como a cortada é executada em pouco tempo e os elementos possuem forte interdependên- 
cia, esse conceito de transferência da parte para o todo parece ser pouco aplicável. Entretanto, alguns professores o utilizaram, pois talvez tenham optado por tornar a organização da cortada menos forte. Isso é justificável, pois aprendizes iniciantes apresentam dificuldade para dirigir atenção a aspectos de interdependência entre elementos de uma habilidade (SCHMIDT; LEE, 2011). Assim, quanto menor a exigência de interação, maior atenção pode ser alocada à consistência dos componentes. Apenas mais à frente no processo de aprendizagem é que se recomenda aumentar a quantidade de fontes para as quais a atenção deverá ser dirigida (PUBLIO; TANI, 1993).

É importante ressaltar que os argumentos citados nesta discussão permitem apenas indicar algumas especulações sobre qual método deve ser recomendado no processo ensino-aprendizagem da cortada, já que o trabalho possui limitações como a dimensão reduzida da amostra e o foco na opinião dos professores - e não na avaliação e comparação do aprimoramento técnico obtido com cada método. Em síntese, os achados do presente estudo apontam na direção que o processo ensinoaprendizagem da cortada deve ser desenvolvido com a introdução de algum tipo de fracionamento. Além disso, a escolha do método de ensino da cortada parece guardar relação com o tempo de experiência do professor de EF: os mais experientes preferem adotar o método combinado entre todo e partes, enquanto os iniciantes preferem o método por partes progressivas.

Part and whole techniques for the volleyball spike from the perspective of Physical Education teachers

\begin{abstract}
Given that the use of part and whole techniques for the volleyball spike has generated a great deal of theoretical and practical debate, the purpose of the present study was to investigate which methods Physical Education teachers use in different professional phases in order to teach the volleyball spike. Moreover, this study was aimed at describing the reasons why these methods were chosen. Thirty six Physical Education teachers with different career levels responded to a questionnaire on the use of teaching methods regarding part and whole techniques for teaching the spike. Higher frequencies of responses for part techniques were detected in teachers at the beginning of their careers, who claimed that task complexity was the main reason for doing so.
\end{abstract}

Keywords: Methods, Teaching, Volleyball, Physical Education and Training. 
Los métodos de fraccionamiento del remate de voleibol desde la perspectiva de profesores de Educación Física

\section{Resumen}

Debido a que la práctica de remate de voleibol con el fraccionamiento ser un tema controvertido y ha generado muchas las inconsistencias teóricas y prácticas, el propósito de este estudio fue investigar cuales dos métodos de fraccionamiento del remate son utilizadas por los profesores de Educación Física en las diferentes etapas profesionales en el proceso de enseñanza y el aprendizaje del remate. Además, hemos tratado de describir la justificación de docentes para selección de uno de los métodos. La muestra se compone de 36 profesores de educación física con los niveles de experiencia diferente, que responderán a un cuestionario sobre el uso de métodos de enseñanza para el fraccionamiento del remate de voleibol. Las frecuencias más altas de respuestas favorables a el fraccionamiento fueran dadas por los profesores en niveles iniciales de la carrera, que justificaram la complejidad del movimiento como la razón principal para el empleo de tal procedimiento.

Palabras clave: Métodos. Enseñanza. Voleibol. Educación y Entrenamiento Físico.

\section{Referências}

BAECKER, I.M. Método parcial e método global: Um estudo comparativo da aprendizagem do quipe de cabeça no solo e da saída da barra fixa em sublance. Revista Kinesis, Santa Maria, p. 63-80, Número Especial (dez.), 1984.

BERNILER, D.C. In pursuit of the expert pedagogue. Educational Researcher, v. 15, n. 7, p. 5-13, 1986.

BEZAULT, P. O voleibol. Lisboa: Editorial Estampa, 2002.

BIZZOCHI, C. O voleibol de alto nível: da iniciação à competição. São Paulo: Fazendo Arte, 2000.

BOJIKIAN, J.C.M. Ensinando voleibol. Guarulhos: Phorte, 1999.

CANFIELD, J.T. Aprendizagem Motora. Santa Maria: Imprensa Universitária, 1981.

CANFIELD, J.T.; REIS C. Aprendizagem motora no voleibol. Santa Maria: JTC, 1998.

CARVALHO, O.M. Voleibol moderno. Brasília: MEC, 1980. 
CASCO, P. Vôlei. São Paulo: Odysseus, 2007.

CORREAA, U.C.; SILVA, A.S.; PAROLI, R. Efeitos de diferentes métodos de ensino na aprendizagem do futebol de salão. Motriz, Rio Claro, v. 10, n. 2, p. 79-88, 2004.

COSTA, S.S.C.; MOREIRA, M.A. Resoluções de problemas I: diferenças entre novatos e especialistas. Investigações em Ensino de Ciências, Porto Alegre, v. 1, n. 2, p. 176-192, 1996.

DUTRA, L. et al. Prática pela parte e pelo todo na aprendizagem da cortada no voleibol. Revista Brasileira de Educação Física e Esporte, São Paulo, v. 20, n. 5, p. 213-214, 2006 a.

DUTRA, L. et al. Prática pelo todo e por adição na aprendizagem da cortada do voleibol. Revista Brasileira de Educação Física e Esporte, São Paulo, v. 20, n. 5, p. 220-221, 2006 b.

FONTANA, F.E. et al. Whole and part practice: A meta-analysis. Perceptual and Motor Skills, 109, p.517-530, 2009.

GOMES, F.R.F. Aprendizagem do Kuzushi (desequilíbio) nos golpes de judô o Soto Gari e Tai Otoshi. 2007. 67f. Dissertação (Mestrado em Pedagogia do Movimento Humano)-Escola de Educação Física e Esporte, Universidade de São Paulo, São Paulo, 2007.

HIDD, F.S. Método de ensino parcial e método de ensino das partes progressivas na aprendizagem do nado crawl. 1991. 130f. Dissertação (Mestrado em Biodinâmica do Movimento Humano)-Escola de Educação Física, Universidade de São Paulo, São Paulo, 1991.

HUBERMAN, M. O ciclo da vida profissional dos professores. In: NÓVOA, A. Vidas de professores. Lisboa: Porto Editora, p.141-169, 1992.

JANUÁRIO, C. Do pensamento do professor à sala de aula. Coimbra: Edições Livraria Almedina, 1996.

KNAPP, B. Skill in sport. London: Routledge \& Kegan, 1970. 
KRUG, A.; KRUG, D.H.F. Métodos de ensino global, analítico e misto na aprendizagem da cortada no voleibol. Relatório de Pesquisa (COPED/CNPq), 1983.

MACHADO, A.A. Voleibol: do aprender ao especializar. Rio de Janeiro: Guanabara Koogan, 2006.

MAGILL, R. Motor learning: Concepts and applications. New York: McGraw-Hill, 2011.

MURRAY, J.F. Effects of whole vs. part method of training on transfer of leraning. Perceptual and Motor Skills, v. 53, n. 3, p. 883-889, 1981.

MOREIRA, C,M,; PEREIRA, J.M. O ensino do conteúdo esporte na escola: o olhar dos professores iniciantes e professores experientes. In: SEMINÁRIO DE ESTUDOS EM EDUCAÇÃO FÍSICA ESCOLAR, 2., 2008, São Carlos. Anais... São Carlos: [s. ed.], p. 33-60, 2008.

NAYLOR, J.; BRIGGS, G. Effects of task complexity and task organization on the relative efficiency of part and whole training methods. Journal of Experimental Psychology, v. 65, n. 3, p. 217-224, 1963.

PUBLIO, N.S.; TANI, G. Aprendizagem de habilidades motoras seriadas da ginástica olímpica. Revista Paulista de Educação Física, São Paulo, v. 7, n. 1, p. 58-68, 1993.

SCHMIDT, R.A.; LEE, T.D. Motor control and learning. Champaign, Human Kinetics, 2011.

SCHMIDT, R.A.; WRISBERG, C.A. Motor learning and performance: A situation-based learning approach. Champaign, Human Kinetics, 2008.

TANI, G. Cinesiologia, educação física e esporte: ordem emanente do caos na estrutura acadêmica. Motus Corporis, n. 3, p. 9-50, 1996.

THOMAS, J.R.; NELSON, J.K.; SILVERMAN, S.J. Métodos de pesquisa em atividade física. Porto Alegre: ArtMed, 2012.

XAVIER, T.P. Métodos de ensino em Educação Física. São Paulo: Manole, 1986. 
WRISBERG, C. Sport skill instruction for coaches. Champaign: Human Kinetics, 2007.

Recebido em: 04/06/2012

Revisado em: 11/11/2012

Aprovado em: 19/01/2013

\section{Endereço para correspondência}

cmj@usp.br

Cassio Miranda Meira Jr.

Universidade de São Paulo, USP Leste.

Av. Arlindo Bettio 1000

Ermelino Matarazzo

03828000 - Sao Paulo, SP - Brasil 